\title{
Progress in QCD next-to-leading order calculations
}

\author{
Zoltán Trócsányi*† \\ University of Debrecen and \\ Institute of Nuclear Research of the Hungarian Academy of Sciences \\ H-4001 Debrecen, PO Box 51, Hungary \\ E-mail: 'Z̃. Trocsanyi@atomki.hü
}

ABSTRACT: I review progress related to the calculation of QCD jet cross sections at the NLO accuracy. After a short introduction into the theory of NLO calculations, I discuss two recent developments: the calculation of two- and three-jet leptoproduction at the NLO accuracy and the extension of the dipole subtraction method for computing NLO corrections for processes involving massive partons.

Jet cross sections at the NLO accuracy consist of the first two terms of the perturbative expansion in the strong coupling,

$$
\sigma=\sigma^{\mathrm{LO}}+\sigma^{\mathrm{NLO}}=\int_{m} \mathrm{~d} \sigma^{\mathrm{B}}+\sigma^{\mathrm{NLO}} .
$$

The leading order cross section is the integral of the fully exclusive Born matrix element of $m$ final-state partons over the available phase space. The NLO correction is a sum of two terms: the real correction is the integral of the Born matrix element of $m+1$ finalstate partons and the virtual correction is the integral of the interference term between the Born-level and one-loop amplitudes of $m$ final state partons,

$$
\sigma^{\mathrm{NLO}}=\int_{m+1} \mathrm{~d} \sigma^{\mathrm{R}}+\int_{m} \mathrm{~d} \sigma^{\mathrm{V}}
$$

In four dimensions both contributions are divergent. An observable is IR-safe if the total NLO correction is finite in four dimensions. However, for almost all cases of interest the phase-space integrations cannot be performed analytically, therefore, in the sum, the cancellation of the singularities requires special care. In the literature several general methods are described [i] $\left[\begin{array}{l}1 \\ ]\end{array}\right]$ to find this finite contribution, each relying on the same principle, namely we subtract an auxiliary cross section from the real corrections such that $\mathrm{d} \sigma^{\mathrm{A}}$ has the same pontwise singular behaviour in any dimensions as $\mathrm{d} \sigma^{\mathrm{R}}$. Moreover, $\mathrm{d} \sigma^{\mathrm{A}}$ has to be

${ }^{*}$ Speaker.

${ }^{\dagger}$ Széchenyi fellow of the Hungarian Ministry of Education 
chosen simple enough, so that it is analytically integrable in $d$ dimensions over the oneparton subspaces that cause the soft and collinear divergences and thus it can be combined with the virtual contribution to give a finite correction in four dimensions. Thus the total NLO contribution can be written as a sum of two terms, an $(m+1)$-parton and an $m$-parton integral,

$$
\sigma^{\mathrm{NLO}}=\int_{m+1}\left[\left(\mathrm{~d} \sigma^{\mathrm{R}}\right)_{\varepsilon=0}-\left(\mathrm{d} \sigma^{\mathrm{A}}\right)_{\varepsilon=0}\right]+\int_{m}\left[\mathrm{~d} \sigma^{\mathrm{V}}+\int_{1} \mathrm{~d} \sigma^{\mathrm{A}}\right]_{\varepsilon=0} .
$$

Although, the principle is simple, the numerical implementation that is also stable and efficient is difficult. The various methods, that differ in the explicit choice for the auxiliary cross section, were developed with the aim of finding the solution with the best numerical behaviour. In this respect, the dipole method [i, $]$ has several advantageous features, only partially shared by the other techniques: (i) The calculation is exact; the same auxiliary cross section is subtracted and added back. (ii) The phase space is exactly the same for the $\mathrm{d} \sigma^{\mathrm{R}}$ as for $\mathrm{d} \sigma^{\mathrm{A}}$, which allows for an efficient generation of the phase space, most suitable for the actual calculation at hand. (iii) There is no need for any particular manipulation of the squared matrix element. (iv) The calculation is Lorentz invariant at any intermediate step, thus switching between various frames of reference can be achieved by simply transforming the momenta. (v) There is no need for crossing functions, thus partonic cross sections can be calculated also with hadrons in the initial state. (vi) It can be implemented in a fully process independent way, therefore, a truly general purpose code, suitable for any initial states, can be written and the same matrix elements in the same code can be used for various processes. The first such example is the NLOJET++ code that already incorporates three- and four-jet production in $e^{+} e^{-}$annihilation [3] 30 , two- and three-jet leptoproduction

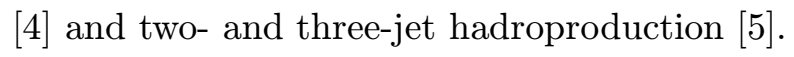

As a new application of the method, let us turn to the computation of NLO corrections to three-jet leptoproduction. Although the NLOJET++ program can be used for calculating any IR-safe observable, for the sake of comparison with existing data I present differential distributions that were also measured by the H1 collaboration at HERA. In order to make the comparisons, I used the same kinematic range as employed in the experiment, i.e., $5 \mathrm{GeV}^{2}<Q^{2}<5000 \mathrm{GeV}^{2}, 0<x_{\mathrm{Bj}}<1,0.2<y<0.6,-1<\eta_{\text {jet }}^{\text {lab }}<2.5, E_{T, \text { jet }}^{\text {Breit }}>5 \mathrm{GeV}$. The hard scale was chosen to be the average transverse momentum of the three-jets. The leading order curves were obtained using the CTEQ5L pdf's and $\alpha_{s}$ run from $\alpha_{s}\left(M_{\mathrm{Z}^{0}}\right)=$ 0.127 using one-loop running, and the NLO curves with CTEQ5M1 pdf's and $\alpha_{s}$ run from $\alpha_{s}\left(M_{\mathrm{Z}^{0}}\right)=0.118$ using two-loop running. The three-jet final states were selected using the inclusive $k_{T}$ algorithm. The plots in Figs. $i_{-1}^{1}$ and ${ }_{2}^{2}$ show the differential distributions in the momentum transfer squared and the Bjorken variable, respectively. We can observe that the leading order predictions have different shape than the data: too low for small values of the variables and too high for large values. The radiative corrections bring theory and experiment much closer. Taking into account the hadronization corrections, the NLO prediction gives a remarkably good description of the data.

We should consider the impressive agreement with caution. For small values of $Q^{2}$ the radiative corrections are rather large (between 30 and $60 \%$ ), so we may expect that the higher order corrections are also large, which is also indicated if we look at the dependence 
$\mathrm{k}_{\perp}$ inclusive

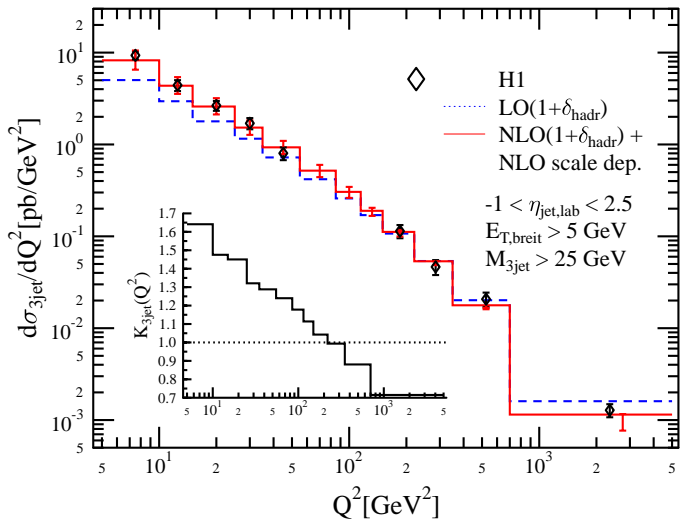

Figure 1: The three-jet differential distribution in the momentum transfer squared $Q^{2}$.

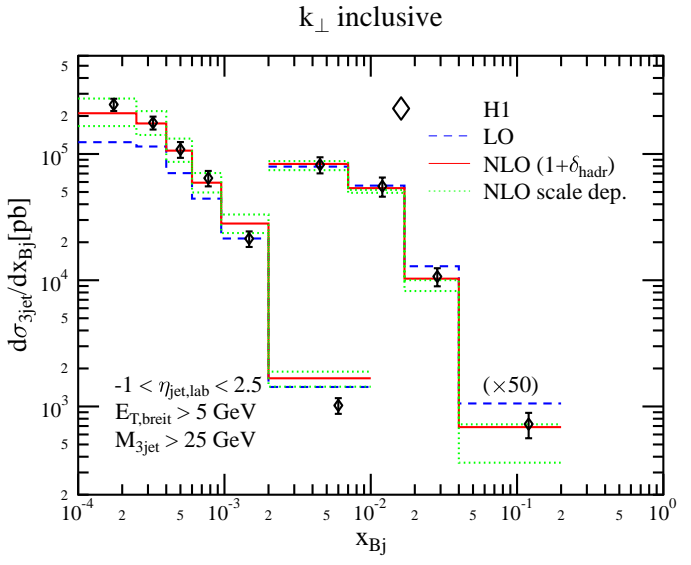

Figure 2: The three-jet differential distribution in the $x_{\mathrm{Bj}}$ Bjorken variable.

of the NLO prediction for the $(3+1)$-jet inclusive cross section on the renormalization and factorization scales in Fig. $\overline{3}$. The scale dependence of the cross section at NLO is still large indicating potentially large higher order corrections. At high $Q^{2}$ (see Fig. 育i we find that the renormalization-scale dependence is reduced significantly, while the originally not too strong factorization-scale dependence does not change substantially. Setting the two scales equal, at NLO we find a rather flat curve with a wide plato around the chosen hard scale. If $Q^{2}$ is large, however, the NLO corrections are negative indicating that all order resummation of terms such as $\ln 1 / x_{\mathrm{Bj}}$ may be important, which will typically increase the cross sections.

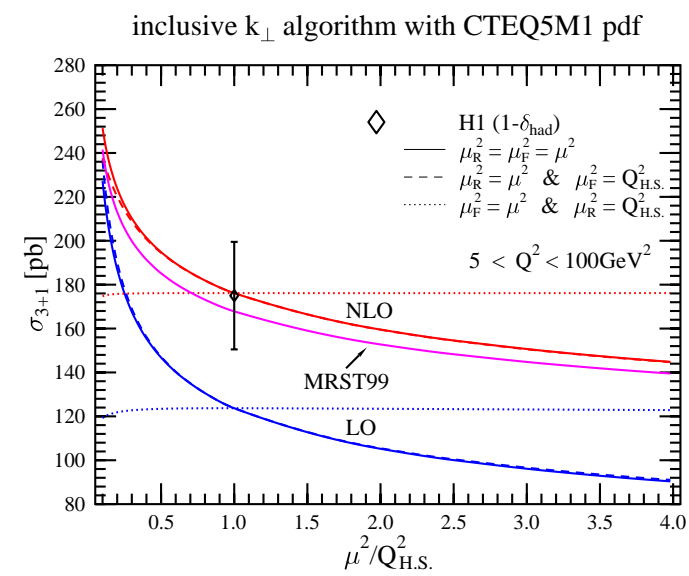

Figure 3: The scale-dependences of the inclusive three-jet cross section at low $Q^{2}$.

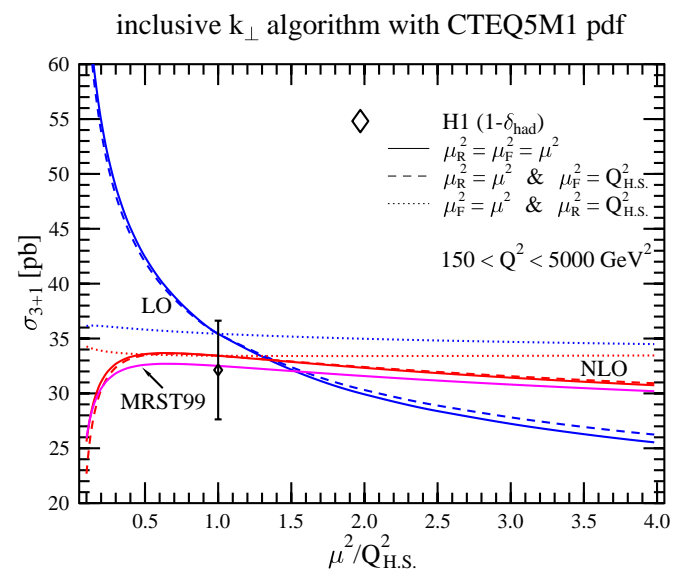

Figure 4: The scale-dependences of the inclusive three-jet cross section at high $Q^{2}$.

Let us now turn to discussing some developments in the theory of NLO calculations for processes involving massive partons. A lot of such calculations exist in the literature, but most of them were carried out for a specific process, not with the general methods worked out for processes of massless partons. The role of massive partons will become even more 
important when the LHC comes into operation and perhaps a lot of new massive coloured particles will be found, or at least will be searched for. Recently, both the slicing [i, $[\bar{i}]$ and the dipole $[\bar{i} \mathbf{i}]$ techniques were extended to taking into account the parton masses. Here I advocate another extension of the dipole method which has a smooth zero-mass limit.

In order to understand why the zero-mass limit may be important, let us recall the different physical roles the finite mass $M$ of the QCD partons play in different physical processes. In some processes (e.g. the total cross section for heavy-quark hadroproduction) the finite (and large) value of $M$ has the essential role of setting the hard scale of the cross section. In these cases the massless limit is IR unstable, and the corresponding cross section cannot be computed in QCD perturbation theory. In other processes (e.g. the production of heavy-flavoured jets), instead, the hard scale $Q$ is independent of the mass $M$, and the latter has only the role of an auxiliary (though important) kinematic scale. These processes are IR stable in the massless limit, that is, when $M \rightarrow 0$ the cross section is still infraredand collinear-safe and, thus, perturbatively computable.

The processes that are perturbatively stable in the massless limit are often studied in kinematic regions where the typical hard scale $Q$ is much larger than the mass $M$ of one (or more) of the heavy partons. In this regime the integral of the real term $\mathrm{d} \sigma^{\mathrm{R}}(M)$ of the NLO cross section in eq. (굴 $)$ leads to contributions of the type

$$
\begin{aligned}
\int_{m+1} \mathrm{~d} \sigma^{\mathrm{R}}(M) & \rightarrow \int_{0}^{Q^{2}} \mathrm{~d} \mathbf{q}_{\perp}^{2}\left(\mathbf{q}_{\perp}^{2}\right)^{-\varepsilon} \frac{1}{\mathbf{q}_{\perp}^{2}+M^{2}} \widetilde{Q \gg M} \ln \frac{Q^{2}}{M^{2}}+\mathrm{O}(\varepsilon), \\
\int_{m+1} \mathrm{~d} \sigma^{\mathrm{R}}(M) & \rightarrow \int_{0}^{Q^{2}} \mathrm{~d} \mathbf{q}_{\perp}^{2}\left(\mathbf{q}_{\perp}^{2}\right)^{-\varepsilon} \frac{M^{2}}{\left[\mathbf{q}_{\perp}^{2}+M^{2}\right]^{2}} \widetilde{Q \gg M} M^{2} \frac{1}{M^{2}}+\mathrm{O}(\varepsilon),
\end{aligned}
$$

where $\mathbf{q}_{\perp}$ generically denotes the typical transverse momentum of the heavy parton with mass $M$. Since these contributions are finite when $\varepsilon \rightarrow 0$, naively, they would not require any special treatments within the subtraction method. However, this could lead to serious numerical problems in kinematic regions where $Q \gg M$. When computing the NLO cross section, the large $\ln Q^{2} / M^{2}$ contribution would appear in the first term (the

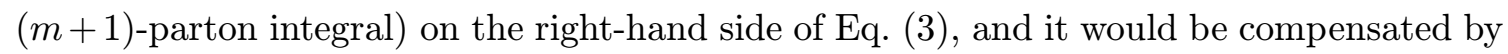
an equally large (but with opposite sign) logarithmic contribution arising from the second term (the $m$-parton integral). Owing to the presence of several large (although compensating) contributions, a similar naive procedure would lead to instabilities in any numerical implementations of the NLO calculation. The numerical instabilities would increase by increasing the ratio $Q / M$ and, in particular, they would prevent from performing the massless limit. The second contribution in Eq. (5.15) can also lead to numerical instabilities due to the presence of a linearly divergent (in the limit $M^{2} / Q^{2} \rightarrow 0$ ) integral, because its variance increases linearly with $Q^{2} / M^{2}$.

These numerical problems can be avoided if we set up our massive-parton formalism by choosing the auxiliary cross section $\mathrm{d} \sigma^{\mathrm{A}}(M)$ in such a way that the following property is fulfilled:

$$
\lim _{M \rightarrow 0} \int_{m+1}\left[\left(\mathrm{~d} \sigma^{\mathrm{R}}(M)\right)_{\varepsilon=0}-\left(\mathrm{d} \sigma^{\mathrm{A}}(M)\right)_{\varepsilon=0}\right]=\int_{m+1}\left[\left(\mathrm{~d} \sigma^{\mathrm{R}}(M=0)\right)_{\varepsilon=0}-\left(\mathrm{d} \sigma^{\mathrm{A}}(M=0)\right)_{\varepsilon=0}\right] .
$$


To avoid the problems related to the large logarithmic contributions in Eq. (言i ficient to impose that the integral of the subtracted cross section on the left-hand side of

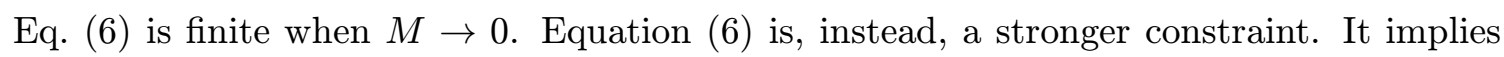
that, in the evaluation of the subtracted cross section, the massless limit (or, more generally, the limit $M / Q \rightarrow 0)$ commutes with the $(m+1)$-parton integral. This guarantees that $\left[\mathrm{d} \sigma^{\mathrm{R}}(M)-\mathrm{d} \sigma^{\mathrm{A}}(M)\right]$ does not contain integrands of the type in Eq. ( $\left.\overline{\underline{p}}_{1}^{\prime}\right)$.

The explicit details of the algorithm will be published soon. Here, I only remark that

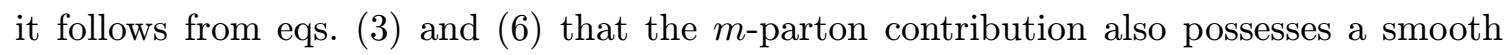
zero-mass limit,

$$
\lim _{M \rightarrow 0} \int_{m}\left[\mathrm{~d} \sigma^{\mathrm{V}}(M)+\int_{1} \mathrm{~d} \sigma^{\mathrm{A}}(M)\right]_{\varepsilon=0}=\int_{m}\left[\mathrm{~d} \sigma^{\mathrm{V}}(M=0)+\int_{1} \mathrm{~d} \sigma^{\mathrm{A}}(M=0)\right]_{\varepsilon=0},
$$

provided the analytic integration over the one-parton phase space, $\int_{1}$ is performed uniformly in the parton masses. Relation $(\overline{\overline{7}})$ can be utilized to deduce the universal singular

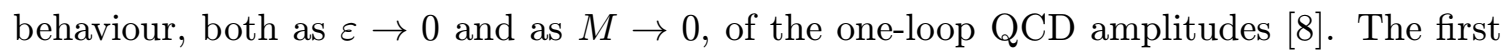
computation employing our techniques is published in Ref. $[\overline{9} \mid \overline{1}]$ ).

In summary, the theory of NLO calculations is well established, including massive partons. For QCD phenomenology the NLO corrections are known for the most interesting processes, including two- and three-jet hadro- and leptoproduction and two-jet photoproduction. Jet phenomenology could benefit from (i) a program for three-jet photoproduction, (ii) programs for heavy-flavoured jet lepto-, hadro-, photoproduction and (iii) programs for vector-boson(s) and associated jet(s) hadroproduction.

I thank my collaborators, Z. Nagy (first part) and S. Catani, S. Dittmaier and M.H. Seymour (second part) for a pleasant a fruitful collaboration. This work was supported in part by the EU Fourth Framework Programme grant FMRX-CT98-0194 (DG 12 - MIHT) and by the Hungarian Scientific Research Fund grant OTKA T-025482.

\section{References}

[1] Proceedings of the Workshop on Standard model physics (and more) at the LHC, Eds. G. Altarelli and M.L. Mangano, CERN-2000-04, Geneva 2000.

[2] S. Catani and M. H. Seymour, Phys. Lett. B 378 (1996) 287 [hep-ph/9602277], Nucl. Phys. B 485 (1997) 291 [Erratum-ibid. B 510 (1997) 291] [hep-ph/9605323].

[3] Z. Nagy and Z. Trócsányi, Phys. Rev. Lett. 79 (1997) 3604 [hep-ph/9707309], Phys. Rev. D 59 (1999) 014020 [Erratum-ibid. D 62 (1999) 099902] [hep-ph/9806317].

[4] Z. Nagy and Z. Trócsányi, Phys. Rev. Lett. 87 (2001) 082001 [hep-ph/0104315].

[5] Z. Nagy, preprint IPPP/01/48 [hep-ph/0110315].

[6] S. Keller and E. Laenen, Phys. Rev. D 59 (1999) 114004 [hep-ph/9812415].

[7] L. Phaf and S. Weinzierl, JHEP 0104 (2001) 006 [hep-ph/0102207].

[8] S. Catani, S. Dittmaier and Z. Trócsányi, Phys. Lett. B 500 (2001) 149 [hep-ph/0011222].

[9] W. Beenakker, S. Dittmaier, M. Kramer, B. Plumper, M. Spira and P. M. Zerwas, Phys. Rev. Lett. 87 (2001) 201805 [hep-ph/0107081]. 